YITP-07-86

\title{
Dynamical D4-D8 and D3-D7 branes in supergravity
}

\author{
Pierre Binetruy \\ Astroparticule et Cosmologie, Université Paris Diderot, CNRS/IN2P3, \\ CEA/DSM, Observatoire de Paris, Bâtiment Condorcet 10, \\ rue Alice Domon et Léonie Duquet, 75205 Paris Cedex 13, France. \\ Misao Sasaki \\ Yukawa Institute for Theoretical Physics Kyoto University, Kyoto 606-8502, Japan. \\ Kunihito Uzawa* \\ Osaka City University Advanced Mathematical Institute, Osaka 558-8585, Japan.
}

(Dated: October 30, 2018)

\begin{abstract}
We present a class of dynamical solutions for intersecting D4-D8 and D3-D7 brane systems in ten-dimensional type IIA and IIB supergravity. We discuss if these solutions can be recovered in lower-dimensional effective theories for the warped compactification of a general p-brane system. It is found that an effective $p+1$-dimensional description is not possible in general due to the entanglement of the transverse coordinates and the $p+1$-dimensional coordinates in the metric components. For the D4-D8 brane system, the dynamical solutions reduces to a static warped $\mathrm{AdS}_{6} \times \mathrm{S}^{4}$ geometry in a certain spacetime region. For the D3-D7 brane system, we find a dynamical solution whose metric form is similar to that of a D3-brane solution. The main difference is the existence of a nontrivial dilaton configuration in the D3-D7 solution. Then we discuss cosmology of these solutions. We find that they behave like a Kasner-type cosmological solution at $\tau \rightarrow \infty$, while it reduces to a warped static solution at $\tau \rightarrow 0$, where $\tau$ is the cosmic time.
\end{abstract}

PACS numbers: 11.25.-w, 11.27.+d, 98.80.Cq

\footnotetext{
*Also at Yukawa Institute for Theoretical Physics Kyoto University, Kyoto 606-8502, Japan.
} 


\section{INTRODUCTION}

Recently, studies on dynamical solutions of supergravity have been a topic of great interest. Conventionally time-dependent solutions of higher-dimensional supergravity are discussed in the context of lower-dimensional effective theories after compactifying the internal space. However, it is unclear how far this effective low-dimensional description is valid. Thus, it is much more desirable to discuss the four-dimensional cosmology in terms of the dynamics of the original higher-dimensional theory. This is particularly true in string cosmology in which the behavior of the early Universe is to be understood in the light of string theory. Indeed, it was pointed out that the four-dimensional effective theory for warped compactification of ten-dimensional type IIB supergravity allows solutions that cannot be obtained from solutions in the original higher-dimensional theories [1].

A time-dependent solution with fiveform flux in the ten-dimensional type IIB supergravity was obtained by Gibbons, Lu and Pope [2]. Requiring the time dependence of the metric for general black $p$-brane systems, it was found that the structure of a warp factor that depends on the time is different from the usual "product type" ansatz [3, 4].

In this paper, we consider dynamical solutions for intersecting brane systems in supergravity, in which gravity is not only coupled to a single gauge field but also to several combinations of scalar and gauge fields. Although the configurations we consider are not directly related to actual warped compactifications to four dimensions, we do so in the hope that they may serve as a first step toward the understanding of realistic, dynamical compactifications that should have occurred in the early Universe.

Some intersecting brane solutions were originally founded by Güven in eleven-dimensional supergravity [5]. A D4-D8 brane system was constructed by Polchinski and Witten using the dual description of the type I string theory on $\mathbb{R}^{9} \times \mathrm{S}^{1}$ with $N$ coinciding D5 branes wrapping the circle [6].

After that, several authors investigated solutions describing intersecting branes, and constructed new static solutions [7, 8]. Nastase analyzed a D4-D8 brane solution as a setup for the holographic dual of QCD [9]. Brandhuber and Oz found a classical solution of intersecting D4-D8 branes [10]. In a certain region of spacetime, the geometry is warped $\mathrm{AdS}_{6} \times \mathrm{S}^{4}$, which describes spontaneous compactification of the massive IIA supergravity in

ten dimensions [11] to the gauged supergravity [12, 13]. In connection with gauge/gravity 
correspondence, a supergravity background of D3-D7 brane intersection has been constructed which includes a flavor D7 brane[14, 15, 16, 17].

The paper is organized as follows. In $\S$ Sec.II, we first consider $p$-brane systems in $D$ dimensions and derive a class of dynamical solutions under a certain metric ansatz. In $\S$ Sec.III, focusing on intersecting D4-D8 brane systems in the ten-dimensional type IIA supergravity, we extend the metric ansatz used in the previous section to intersecting branes and obtain a class of dynamical solutions. Then further specializing the form of the metric, we consider a cosmological solution. Interestingly, this solution is found to approach a warped static solution as $\tau \rightarrow 0$ and a Kasner-type anisotropic solution as $\tau \rightarrow \infty$, where $\tau$ is the cosmic time. We investigate the dynamical D3-D7 brane solution of the ten-dimensional type IIB supergravity in $\S$ Sec.IV. Finally, we conclude in $\S$ Sec.V.

\section{DYNAMICAL $p$-BRANE SOLUTIONS}

In this section, we consider dynamical p-brane systems in $D$ dimensions. First, we write down the Einstein equations under a particular ansatz for the metric, which is a generalization of the metric form of known static $p$-brane solutions. Then, we solve the Einstein equations and present the solutions explicitly.

We consider a gravitational theory with the metric $g_{M N}$, dilaton $\phi$, and an antisymmetric tensor field of rank $(p+2)$. This corresponds to a $p$-brane system in string theory. The most general action for the $p$-brane system in the Einstein frame can be written as [18]

$$
S=\frac{1}{2 \kappa^{2}} \int\left(R * \mathbf{1}_{D}-\frac{1}{2} d \phi \wedge * d \phi-\frac{1}{2} e^{-c \phi} F_{(p+2)} \wedge * F_{(p+2)}\right),
$$

where $\kappa^{2}$ is the $D$-dimensional gravitational constant, $*$ is the Hodge dual operator in the $D$-dimensional spacetime, and $c$ is a constant given by

$$
c^{2}=4-\frac{2(p+1)(D-p-3)}{D-2} .
$$

The expectation values of fermionic fields are assumed to be zero.

After variations with respect to the metric, the dilaton, and the $(p+1)$-form gauge field, 
we obtain the field equations

$$
\begin{aligned}
& R_{M N}=\frac{1}{2} \partial_{M} \phi \partial_{N} \phi \\
& +\frac{1}{2} \frac{1}{(p+2) !} e^{-c \phi}\left[(p+2) F_{M A_{2} \cdots A_{p+2}} F_{N} A_{2} \cdots A_{p+2}-\frac{p+1}{D-2} g_{M N} F_{(p+2)}^{2}\right], \\
& \triangle \phi=-\frac{1}{2} \frac{c}{(p+2) !} e^{-c \phi} F_{(p+2)}^{2}, \\
& d\left(e^{-c \phi} * F_{(p+2)}\right)=0
\end{aligned}
$$

where $\triangle$ is the $D$-dimensional Laplace operator.

To solve the field equations, we assume the $D$-dimensional metric in the form

$$
d s^{2}=h^{a}(x, y) q_{\mu \nu} d x^{\mu} d x^{\nu}+h^{b}(x, y) u_{i j} d y^{i} d y^{j}
$$

where $q_{\mu \nu}$ is a $(p+1)$-dimensional metric, which depends only on the $(p+1)$-dimensional coordinates $x^{\mu}$, and $u_{i j}$ is the $(D-p-1)$-dimensional metric, which depends only on the $(D-p-1)$-dimensional coordinates $y^{i}$. The parameters $a$ and $b$ are given by

$$
a=-\frac{D-p-3}{D-2}, \quad b=\frac{p+1}{D-2} .
$$

The metric form (6] $)$ is a straightforward generalization of the case of a static p-brane system with a dilaton coupling [18]. Furthermore, we assume that the scalar field $\phi$ and the gauge field strength $F_{(p+2)}$ are given by

$$
\begin{aligned}
& e^{\phi}=h^{-c / 2}, \\
& F_{(p+2)}=d\left(h^{-1}\right) \wedge \Omega(\mathrm{X}),
\end{aligned}
$$

where $\Omega(\mathrm{X})$ denotes the volume $(p+1)$ form

$$
\Omega(\mathrm{X})=\sqrt{-q} d x^{0} \wedge d x^{1} \wedge \cdots \wedge d x^{p}
$$

Here, $q$ is the determinant of the metric $q_{\mu \nu}$.

Let us first consider the Einstein Eqs. (3). Using the assumptions (6), (8) and (9), the Einstein equations are given by

$$
\begin{aligned}
& R_{\mu \nu}(\mathrm{X})-h^{-1} D_{\mu} D_{\nu} h-\frac{a}{2} h^{-1} q_{\mu \nu}\left(\triangle_{\mathrm{X}} h+h^{-1} \triangle_{\mathrm{Y}} h\right)=0, \\
& R_{i j}(\mathrm{Y})-\frac{b}{2} u_{i j}\left(\triangle_{\mathrm{X}} h+h^{-1} \triangle_{\mathrm{Y}} h\right)=0, \\
& \partial_{\mu} \partial_{i} h=0,
\end{aligned}
$$


where $D_{\mu}$ is the covariant derivative with respect to the metric $q_{\mu \nu}, \triangle_{\mathrm{X}}$ and $\triangle_{\mathrm{Y}}$ are the Laplace operators on the space of $\mathrm{X}$ and the space $\mathrm{Y}$, and $R_{\mu \nu}(\mathrm{X})$ and $R_{i j}(\mathrm{Y})$ are the Ricci tensors of the metrics $q_{\mu \nu}$ and $u_{i j}$, respectively. From Eq. (13), the warp factor $h$ must be in the form

$$
h(x, y)=h_{0}(x)+h_{1}(y) .
$$

With this form of $h$, the other components of the Einstein Eqs. (11) and (12) are rewritten as

$$
\begin{aligned}
& R_{\mu \nu}(\mathrm{X})-h^{-1} D_{\mu} D_{\nu} h_{0}-\frac{a}{2} h^{-1} q_{\mu \nu}\left(\triangle_{\mathrm{X}} h_{0}+h^{-1} \triangle_{\mathrm{Y}} h_{1}\right)=0 \\
& R_{i j}(\mathrm{Y})-\frac{b}{2} u_{i j}\left(\triangle_{\mathrm{X}} h_{0}+h^{-1} \triangle_{\mathrm{Y}} h_{1}\right)=0 .
\end{aligned}
$$

Let us next consider the gauge field. Under the assumption (9), we find

$$
d F_{(p+2)}=h^{-1}\left(2 \partial_{i} \ln h \partial_{j} \ln h+h^{-1} \partial_{i} \partial_{j} h\right) d y^{i} \wedge d y^{j} \wedge \Omega(\mathrm{X})=0
$$

Thus, the Bianchi identity is automatically satisfied. Also the equation of motion for the gauge field becomes

$$
d\left[e^{-c \phi} * F_{(p+2)}\right]=-d\left[\partial_{i} h\left(*_{\mathrm{Y}} d y^{i}\right)\right]=0,
$$

where $*_{Y}$ denotes the Hodge dual operator on Y. Hence, the gauge field equation is automatically satisfied under the assumption (9).

Let us consider the scalar field equation. Substituting the forms of the scalar field (8), the gauge field (9), and the warp factor (14) into the equation of motion for the scalar field (4), we obtain

$$
\frac{c}{2} h^{-b}\left(\triangle_{\mathrm{X}} h_{0}+h^{-1} \triangle_{\mathrm{Y}} h_{1}\right)=0,
$$

Thus, unless the parameter $c$ is zero, the warp factor $h$ should satisfy the equations

$$
\triangle_{\mathrm{X}} h_{0}=0, \quad \triangle_{\mathrm{Y}} h_{1}=0
$$

If $F_{(p+2)} \neq 0$, the function $h_{1}$ is nontrivial. In this case, the Einstein equations reduce to

$$
\begin{aligned}
& R_{\mu \nu}(\mathrm{X})=0, \\
& R_{i j}(\mathrm{Y})=0, \\
& D_{\mu} D_{\nu} h_{0}=0 .
\end{aligned}
$$


On the other hand, if $F_{(p+2)}=0$, the function $h_{1}$ becomes trivial. Namely, the internal space is no longer warped [1].

As a special example, we consider the case

$$
q_{\mu \nu}=\eta_{\mu \nu}, \quad u_{i j}=\delta_{i j}
$$

where $\eta_{\mu \nu}$ is the $(p+1)$-dimensional Minkowski metric and $\delta_{i j}$ is the $(D-p-1)$-dimensional Euclidean metric. In this case, the solution for $h$ can be obtained explicitly as

$$
h(x, y)=A_{\mu} x^{\mu}+B+\sum_{l} \frac{M_{l}}{\left|y^{i}-y_{l}^{i}\right|^{D-p-3}},
$$

where $A_{\mu}, B$, and $M_{l}$ are constant parameters. In the case of $D=11, p=2$, the solution describes an M2 brane [19, 20].

Here, we mention an important fact about the nature of the dynamical solutions described in the above. In general, we regard the $(p+1)$-dimensional spacetime to contain our fourdimensional universe, while the remaining space is assumed to be compact and sufficiently small in size. Then one would usually think that an effective $(p+1)$-dimensional description of the theory should be possible at low energies. However, solutions of the above set of equations have the property that they are genuinely $D$ dimensional in the sense that one can never neglect the dependence on $\mathrm{Y}$, say of $h$. This is clear from an inspection of Eqs. (15), (16). In particular, the second equation involves the Laplacian of $h$ with respect to the space X. Hence, the equations determining the internal space Y cannot be determined independently from the geometry of the space $\mathrm{X}$. The origin of this property is due to the existence of a nontrivial gauge field strength, which forces the function $h$ to be a linear combination of a function of $x^{\mu}$ and a function of $y^{i}$, instead of a product of these two types of functions as conventionally assumed. This fact is in sharp contrast with the case when one is allowed to integrate out the internal space to obtain an effective lower-dimensional theory.

Finally, we comment on the exceptional case of $c=0$, which happens when $(D, p)=$ $(10,3),(11,5),(11,2)$. The scalar field becomes constant because of the ansatz (8)), and the scalar field Eq. (19) is automatically satisfied. Then, the Einstein equations become

$$
\begin{aligned}
& R_{\mu \nu}(\mathrm{X})=0 \\
& R_{i j}(\mathrm{Y})=\frac{b}{2}(p+1) \lambda u_{i j}(\mathrm{Y}) \\
& D_{\mu} D_{\nu} h_{0}=\lambda q_{\mu \nu}(\mathrm{X})
\end{aligned}
$$


where $\lambda$ is a constant. As seen from these equations, the internal space $\mathrm{Y}$ is not necessarily Ricci flat, and the function $h_{0}$ becomes more complicated. For example, when the metric $q_{\mu \nu}$ is Minkowski, $h_{0}$ is no longer linear in the coordinates $x^{\mu}$ but quadratic in them [21].

Before concluding this section, let us study the special case of $(D, p)=(10,8)$ as a warmup exercise for the next section. The existence of the D8 brane means the appearance of the 9-form gauge potential, hence the 10-form gauge field strength, which is essentially a cosmological constant. Then the ten-dimensional action in the Einstein frame is given as [11]

$$
S=\frac{1}{2 \kappa^{2}} \int\left(R * \mathbf{1}-\frac{1}{2} d \phi \wedge * d \phi-\frac{1}{2} e^{5 \phi / 2} m^{2} * \mathbf{1}\right)
$$

where $m$ is a constant parameter, which is the dual of the 10 -form field strength $F_{(10)}$ in the string frame. This action is given by the truncations of Romans' massive type IIA supergravity [11, 22, 23], 24].

The field equations are expressed as

$$
\begin{aligned}
\triangle \phi & =\frac{5}{4} e^{5 \phi / 2} m^{2} \\
R_{M N} & =\frac{1}{2} \partial_{M} \phi \partial_{N} \phi+\frac{m^{2}}{16} e^{5 \phi / 2} g_{M N},
\end{aligned}
$$

where $\triangle$ is the ten-dimensional Laplace operator. The ansatz for the metric (6) and the scalar field (8) become

$$
\begin{aligned}
d s^{2} & =h^{1 / 8}(x, y) q_{\mu \nu}(\mathrm{X}) d x^{\mu} d x^{\nu}+h^{9 / 8}(x, y) d y^{2} \\
e^{\phi} & =h^{-5 / 4} .
\end{aligned}
$$

The field equations give

$$
\begin{aligned}
& R_{\mu \nu}(\mathrm{X})=0 \\
& h(x, y)=h_{0}(x) \pm m\left(y-y_{0}\right)
\end{aligned}
$$

where $y_{0}$ is a constant parameter, and $h_{0}(x)$ has to satisfy the equation

$$
D_{\mu} D_{\nu} h_{0}=0
$$

Now we show a solution for $h_{0}$ and $q_{\mu \nu}(\mathrm{X})$ to (23), (28) except for X being $(p+1)$ dimensional Minkowski spacetime. Let us consider a simple gravitational plane-wave metric

$$
q_{\mu \nu}(\mathrm{X}) d x^{\mu} d x^{\nu}=d u d v-K(u, x) d u^{2}+\delta_{i j} d x^{i} d x^{j}
$$


where $(i, j)=(2, \cdots, p+1)$. This is a vacuum solution of the Einstein equations if the function $K$ satisfies

$$
R_{u u}(\mathrm{X})=\frac{1}{2} \partial_{i} \partial^{i} K=0 .
$$

Particular solutions are $K_{1}=a_{i j}(u) x^{i} x^{j}$ with $a_{i i}=0$, or $K_{2}=f(u)|x|^{-p+3}$ for $x \neq 0$.

First, we consider the $c \neq 0$ case in which $D_{v} h_{0}=D_{i} h_{0}=0$. In this case, Eq. (23) is

$$
\partial_{u}^{2} h_{0}=0
$$

Then, the form of $h_{0}$ is given by

$$
h_{0}(u)=c_{1} u+c_{2}
$$

where $c_{1}$ and $c_{2}$ are constant parameters. The metric of $D$-dimensional spacetime can be written as

$$
d s^{2}=\left(c_{1} u+c_{2}+h_{1}\right)^{a} q_{\mu \nu}(\mathrm{X}) d x^{\mu} d x^{\nu}+\left(c_{1} u+c_{2}+h_{1}\right)^{b} u_{i j}(\mathrm{Y}) d y^{i} d y^{j}
$$

where $h_{1}$ satisfies the equation $\triangle_{\mathrm{Y}} h_{1}=0$. Next, for the case $c=0$ in which $D_{v} h_{0}=D_{i} h_{0}=$ 0, Eq. (28) becomes

$$
\partial_{u}^{2} h_{0}=\lambda
$$

The solution of $h_{0}$ is

$$
h_{0}(u)=\frac{\lambda}{2} u^{2}+c_{1} u+c_{2}
$$

where $c_{1}$ and $c_{2}$ are constant parameters. The solution (43) leads to the metric of $D$ dimensional spacetime

$$
d s^{2}=\left(\frac{\lambda}{2} u^{2}+c_{1} u+c_{2}+h_{1}\right)^{a} q_{\mu \nu}(\mathrm{X}) d x^{\mu} d x^{\nu}+\left(\frac{\lambda}{2} u^{2}+c_{1} u+c_{2}+h_{1}\right)^{b} u_{i j}(\mathrm{Y}) d y^{i} d y^{j}
$$

Here, we mention that in the limit when the terms with $h_{1}$ dominates in the metric the whole $D$-dimensional metric becomes static. This is the metric of the static $p$-brane solution [18], which is the so-called warped compactification. On the other hand, in the limit when the terms with $h_{1}$ are negligible, the background changes from the above description to ordinary Kaluza-Klein compactification as time evolves. Although this solution cannot describe a realistic cosmology, it is interesting to note that this cosmological solution is an asymptotically static $p$-brane solution. 


\section{DYNAMICAL SOLUTIONS FOR THE D4-D8 BRANE SYSTEM}

Now we consider dynamical solutions for the D4-D8 brane system, which appears in the ten-dimensional type IIA supergravity. The bosonic action of D4-D8 brane system in the Einstein frame is given by [7, 9, 10, 25]

$$
S=\frac{1}{2 \kappa^{2}} \int\left(R * \mathbf{1}-\frac{1}{2} d \phi \wedge * d \phi-\frac{1}{2 \cdot 4 !} e^{\phi / 2} F_{(4)} \wedge * F_{(4)}-\frac{1}{2} e^{5 \phi / 2} m^{2} * \mathbf{1}\right) .
$$

The equations of motion for $\phi$ and $F_{(4)}$, and the Bianchi identity for $F_{(4)}$ are

$$
\begin{aligned}
& \triangle \phi=\frac{1}{4}\left(5 m^{2} e^{5 \phi / 2}+\frac{1}{4 !} e^{\phi / 2} F_{(4)}^{2}\right), \\
& d\left(e^{\phi / 2} * F_{(4)}\right)=0 \\
& d F_{(4)}=0
\end{aligned}
$$

where $\triangle$ is the ten-dimensional Laplace operator. The Einstein equations are

$$
R_{M N}=\frac{1}{2} \partial_{M} \phi \partial_{N} \phi+\frac{1}{16} m^{2} e^{5 \phi / 2} g_{M N}+\frac{1}{2 \cdot 4 !} e^{\phi / 2}\left(4 F_{M A B C} F_{N}^{A B C}-\frac{3}{8} g_{M N} F_{(4)}^{2}\right) \text {. }
$$

In the following, we look for a solution whose spacetime metric has the form

$$
\begin{aligned}
& d s^{2}=h^{1 / 12}(z)\left[h_{4}^{-3 / 8}(x, r, z) d s^{2}\left(\mathrm{X}_{5}\right)+h_{4}^{5 / 8}(x, r, z)\left(d r^{2}+r^{2} d s^{2}\left(\mathrm{Y}_{3}\right)+d z^{2}\right)\right] \\
& d s^{2}\left(\mathrm{X}_{5}\right)=q_{\mu \nu} d x^{\mu} d x^{\nu} \\
& d s^{2}\left(\mathrm{Y}_{3}\right)=u_{i j} d y^{i} d y^{j}
\end{aligned}
$$

where $q_{\mu \nu}$ is the five-dimensional metric depending only on the coordinates $x^{\mu}$ of $\mathrm{X}_{5}$, and $u_{i j}$ is the three-dimensional metric depending only on the coordinates $y^{i}$ of $\mathrm{Y}_{3}$. As for the scalar field and the 4-form field strength, we adopt the following assumptions:

$$
\begin{aligned}
e^{\phi} & =h^{-5 / 6} h_{4}^{-1 / 4} \\
F_{(4)} & =e^{-\phi / 2} *\left[d\left(h_{4}^{-1}\right) \wedge \Omega\left(\mathrm{X}_{5}\right)\right],
\end{aligned}
$$

where $\Omega\left(\mathrm{X}_{5}\right)$ is given by

$$
\Omega\left(\mathrm{X}_{5}\right)=\sqrt{-q} d x^{0} \wedge d x^{1} \wedge d x^{2} \wedge d x^{3} \wedge d x^{4}
$$

Let us first consider the Einstein Eqs. (499). Under the assumptions (150), (53) and (54), 
the Einstein equations become

$$
\begin{gathered}
R_{\mu \nu}\left(\mathrm{X}_{5}\right)-h_{4}^{-1} D_{\mu} D_{\nu} h_{4}+q_{\mu \nu} h_{4}^{-1}\left[\frac{3}{16} \triangle_{\mathrm{X}_{5}} h_{4}+\frac{3}{16} h_{4}^{-1} \partial_{r}^{2} h_{4}-\frac{1}{24} h^{-1} \partial_{z}^{2} h+\frac{3}{16} h_{4}^{-1} \partial_{z}^{2} h_{4}\right. \\
\left.+\frac{1}{36}\left(\partial_{z} \ln h\right)^{2}-\frac{m^{2}}{16} h^{-2}+\frac{1}{16} \partial_{z} \ln h \partial_{z} \ln h_{4}+\frac{9}{16 r} \partial_{r} \ln h_{4}\right]=0 \\
\partial_{\mu} \partial_{r} h_{4}=0 \\
\partial_{\mu} \partial_{z} h_{4}=0 \\
\triangle_{\mathrm{X}_{5}} h_{4}+h_{4}^{-1}\left[\partial_{r}^{2} h_{4}+\frac{3}{r} \partial_{r} h_{4}+\partial_{z}^{2} h_{4}+\frac{1}{3} \partial_{z} \ln h \partial_{z} h_{4}\right]+\frac{2}{15} h^{-1} \partial_{z}^{2} h \\
+\frac{1}{5}\left[\frac{4}{9}\left(\partial_{z} \ln h\right)^{2}-m^{2} h^{-2}\right]=0, \\
R_{i j}\left(\mathrm{Y}_{3}\right)-2 u_{i j}-\frac{5}{16} r^{2} u_{i j}\left[\triangle_{\mathrm{X}_{5}} h_{4}+h^{-1} \partial_{r}^{2} h_{4}+\frac{3}{r} \partial_{r} \ln h_{4}+h_{4}^{-1} \partial_{z}^{2} h_{4}\right. \\
\left.+\frac{1}{3} \partial_{z} \ln h \partial_{z} \ln h_{4}+\frac{1}{24} h^{-1} \partial_{z}^{2} h+\frac{1}{36}\left(\partial_{z} \ln h\right)^{2}-\frac{m^{2}}{16} h^{-2}\right]=0
\end{gathered}
$$

where $R_{\mu \nu}\left(\mathrm{X}_{5}\right), R_{i j}\left(\mathrm{Y}_{3}\right)$ are the Ricci tensors of the metric $q_{\mu \nu}$ and $u_{i j}$, respectively, $D_{\mu}$ is the covariant derivative with respective to the metric $q_{\mu \nu}$, and $\triangle_{\mathrm{X}_{5}}$ is the Laplace operator on the space $\mathrm{X}_{5}$. From Eqs. (57) and (58), the warp factor $h_{4}$ can be written as

$$
h_{4}(x, y, z)=H_{0}(x)+H_{1}(r, z) .
$$

Inserting this into Eqs. (156), (59), and (60), we find

$$
\begin{aligned}
& R_{\mu \nu}\left(\mathrm{X}_{5}\right)-h_{4}^{-1} D_{\mu} D_{\nu} H_{0}+\frac{3}{16} h_{4}^{-1} q_{\mu \nu}\left[\frac{1}{3}\left(\frac{4}{9}\left(\partial_{z} \ln h\right)^{2}-\frac{2}{9} h^{-1} \partial_{z}^{2} h-m^{2} h^{-2}\right)\right. \\
& \left.+\triangle_{\mathrm{X}_{5}} H_{0}+h_{4}^{-1}\left(\partial_{r}^{2} H_{1}+\frac{3}{r} \partial_{r} H_{1}+\partial_{z}^{2} H_{1}+\frac{1}{3} \partial_{z} \ln h \partial_{z} H_{1}\right)\right]=0, \\
& \triangle_{\mathrm{X}_{5}} H_{0}+h_{4}^{-1}\left(\partial_{r}^{2} H_{1}+\frac{3}{r} \partial_{r} H_{1}+\partial_{z}^{2} H_{1}+\frac{1}{3} \partial_{z} \ln h \partial_{z} H_{1}\right) \\
& +\frac{1}{5}\left(\frac{4}{9}\left(\partial_{z} \ln h\right)^{2}+\frac{2}{3} h^{-1} \partial_{z}^{2} h-m^{2} h^{-2}\right)=0, \\
& R_{i j}\left(\mathrm{Y}_{3}\right)-2 u_{i j}-\frac{5}{16} r^{2} u_{i j}\left[\triangle_{\mathrm{X}_{5}} H_{0}+h_{4}^{-1}\left(\partial_{r}^{2} H_{1}+\frac{3}{r} \partial_{r} H_{1}+\partial_{z}^{2} H_{1}+\frac{1}{3} \partial_{z} \ln h \partial_{z} H_{1}\right)\right. \\
& \left.\quad+\frac{1}{16}\left(\frac{4}{9}\left(\partial_{z} \ln h\right)^{2}+\frac{2}{3} h^{-1} \partial_{z}^{2} h-m^{2} h^{-2}\right)\right]=0 .
\end{aligned}
$$

Let us next consider the gauge field equations. The gauge field Eq. (47) is automatically satisfied under the assumption (54) and the form of $h_{4}$ given by Eq. (61). Under the 
assumptions (50) and (53), (154), the Bianchi identity (48) gives

$$
\begin{aligned}
& \partial_{r}^{2} h_{4}+\frac{3}{r} \partial_{r} h_{4}+\partial_{z}^{2} h_{4}+\frac{1}{3} \partial_{z} \ln h \partial_{z} h_{4}=0, \\
& \partial_{\mu} \partial_{r} h_{4}=0 \\
& \partial_{\mu} \partial_{z} h_{4}=0 .
\end{aligned}
$$

The last two equations are the same as Eqs. (57) and (58), and they have been already solved to give Eq. (61). Then the first Eq. (65) becomes

$$
\partial_{r}^{2} H_{1}+\frac{3}{r} \partial_{r} H_{1}+\partial_{z}^{2} H_{1}+\frac{1}{3} \partial_{z} \ln h \partial_{z} H_{1}=0 .
$$

Next we consider the scalar field Eq. (46). Substituting the assumptions for the metric (50), the scalar and gauge fields (53), (54), and the form of $h_{4}$ (61) into the scalar field Eq. (46), we find

$$
\begin{aligned}
h^{-1 / 12} h_{4}^{-5 / 8} & {\left[\triangle_{\mathrm{X}_{5}} H_{0}+\frac{5}{9}\left(\partial_{z} \ln h\right)^{2}-\frac{5}{4} m^{2} h^{-2}+\frac{5}{6} h^{-1} \partial_{z}^{2} h\right.} \\
+ & \left.h_{4}^{-1}\left(\partial_{r}^{2} H_{1}+\frac{3}{r} \partial_{r} H_{1}+\partial_{z}^{2} H_{1}+\frac{1}{3} \partial_{z} \ln h \partial_{z} H_{1}\right)\right]=0 .
\end{aligned}
$$

Together with Eq. (68), the above equation gives

$$
\triangle_{\mathrm{X}_{5}} H_{0}+\frac{5}{4}\left(\frac{4}{9}\left(\partial_{z} \ln h\right)^{2}+\frac{2}{3} h^{-1} \partial_{z}^{2} h-m^{2} h^{-2}\right)=0 .
$$

Inserting Eqs. (68) and (70) into the Einstein Eqs. (62) - (64), we find for nontrivial $H_{1}$,

$$
\begin{aligned}
& R_{\mu \nu}\left(\mathrm{X}_{5}\right)=0, \\
& R_{i j}\left(\mathrm{Y}_{3}\right)=2 u_{i j}, \\
& D_{\mu} D_{\nu} H_{0}=0, \quad \triangle_{\mathrm{X}_{5}} H_{0}=0, \\
& \frac{4}{9}\left(\partial_{z} h\right)^{2}-m^{2}=0, \quad \partial_{z}^{2} h=0 .
\end{aligned}
$$

The last line of the above equations is immediately solved to give

$$
h(z)=\frac{3}{2} m\left(z-z_{0}\right)
$$

where $z_{0}$ is an integration constant (corresponding to the position of the D8 brane). Below we set $z_{0}=0$ without loss of generality. Then Eq. (68) reduces to

$$
\partial_{r}^{2} H_{1}+\frac{3}{r} \partial_{r} H_{1}+\partial_{z}^{2} H_{1}+\frac{1}{3 z} \partial_{z} H_{1}=0 .
$$


The solution is

$$
H_{1}(r, z)=\frac{c_{1}}{\left(r^{2}+z^{2}\right)^{5 / 3}}+c_{2}
$$

where $c_{1}$ and $c_{2}$ are constant parameters.

Let us investigate the geometrical properties of the D4-D8 brane system. As a particular solution to the three-dimensional metric $u_{i j}$ which satisfies Eq. (72), we take the space $\mathrm{Y}_{3}$ to be a three-dimensional sphere $\mathrm{S}^{3}$. Then if we make a change of coordinates, $z=\tilde{r} \sin \alpha$, $r=\tilde{r} \cos \alpha(0 \leq \alpha \leq \pi / 2)$, the metric reads

$$
d s^{2}=h^{1 / 12}\left[h_{4}^{-3 / 8} q_{\mu \nu} d x^{\mu} d x^{\nu}+h_{4}^{5 / 8}\left(d \tilde{r}^{2}+\tilde{r}^{2} d \Omega_{4}^{2}\right)\right]
$$

where

$$
\begin{aligned}
& d \Omega_{4}^{2}=d \alpha^{2}+\cos ^{2} \alpha d \Omega_{3}^{2}, \\
& h_{4}(x, \tilde{r})=H_{0}(x)+\frac{c_{1}}{\tilde{r}^{10 / 3}}+c_{2}=H_{0}(x)+H_{1}(\tilde{r}), \\
& h(\tilde{r}, \alpha)=\frac{3}{2} m \tilde{r} \sin \alpha .
\end{aligned}
$$

Here, $d \Omega_{3}^{2}$ and $d \Omega_{4}^{2}$ denote the line elements of the three-dimensional sphere $S^{3}$ and the four-dimensional sphere $\mathrm{S}^{4}$, respectively.

Now we further define a new coordinate $U$ by $\tilde{r}^{2}=U^{3}$. From Eq. (73), we see that $H_{0}$ is a linear function of $x^{\mu}$. Hence, keeping the values of these coordinates finite, the metric in the limit $U \rightarrow 0$ becomes

$$
d s^{2}=\left(\frac{3}{2} m \sin \alpha\right)^{1 / 12}\left[c_{1}^{-1 / 2} U^{2} q_{\mu \nu} d x^{\mu} d x^{\nu}+c_{1}^{1 / 2}\left(\frac{9 d U^{2}}{4 U^{2}}+d \Omega_{4}^{2}\right)\right],
$$

while the dilaton is given by

$$
e^{\phi}=c_{1}^{-1 / 4}\left(\frac{3}{2} m \sin \alpha\right)^{-5 / 6} .
$$

This is a static metric. In particular, in the case $q_{\mu \nu}$ is the five-dimensional Minkowski metric $\eta_{\mu \nu}$, the above ten-dimensional metric becomes a warped $\mathrm{AdS}_{6} \times \mathrm{S}^{4}$ space [10, 13, 26, 27].

Let us consider the case $q_{\mu \nu}=\eta_{\mu \nu}$ in more detail. In this case, a solution for the warp factors $h_{4}$ and $h$ can be obtained explicitly as

$$
\begin{aligned}
h_{4}(x, \tilde{r})=\beta t & +K, \\
K & \equiv \quad \beta_{a} x^{a}+\gamma+H_{1}(\tilde{r}), \\
h(\tilde{r}, \alpha) & =\frac{3}{2} m \tilde{r} \sin \alpha,
\end{aligned}
$$


where $x^{a}(a=1, \cdots, 4)$ denote the spatial coordinates of $\mathrm{X}_{5}, H_{1}(\tilde{r})$ is given by (77), and $\beta$, $\beta_{a}$ and $\gamma$ are constant parameters.

Now we introduce a new time coordinate $\tau$ by

$$
\frac{\tau}{\tau_{0}}=(\beta t)^{13 / 16}, \quad \beta \tau_{0}=\frac{16}{13}
$$

where we have assumed $\beta>0$ for simplicity. Then, the ten-dimensional metric (78) is given by

$$
\begin{aligned}
d s^{2}= & h^{1 / 12}\left(1+\left(\frac{\tau}{\tau_{0}}\right)^{-16 / 13} K\right)^{-3 / 8}\left[\left(-d \tau^{2}+\left(\frac{\tau}{\tau_{0}}\right)^{-6 / 13} \delta_{a b} d x^{a} d x^{b}\right)\right. \\
& \left.+\left(1+\left(\frac{\tau}{\tau_{0}}\right)^{-16 / 13} K\right)^{5 / 8}\left(\frac{\tau}{\tau_{0}}\right)^{10 / 13}\left(d \tilde{r}^{2}+\tilde{r}^{2} d \Omega_{4}^{2}\right)\right]
\end{aligned}
$$

where the metric $\delta_{a b}$ is the spatial part of the five-dimensional Minkowski metric $\eta_{\mu \nu}$. If we set $K=0$, the scale factor of the four-dimensional space is proportional to $\tau^{-6 / 13}$, while that for the remaining five-dimensional space is proportional to $\tau^{10 / 13}$. Thus, in the limit when the terms with $K$ are negligible, which is realized in the limit $\tau \rightarrow \infty$, we have a Kaluza-Klein type cosmological solution. Again, similar to the case of the $p$-brane solution discussed in Sec. II, although this solution is by no means realistic, it is interesting to note that it is asymptotically static in the past $\tau \rightarrow 0$.

\section{DYNAMICAL D3-D7 BRANE SOLUTION}

In this section, we consider dynamical solutions for the D3-D7 brane system, which appears in the ten-dimensional type IIB supergravity [28].

\section{A. D7-brane solution}

First, we discuss a D7-brane solution [29]. The action for the D7-brane system in the Einstein frame can be written as

$$
S=\frac{1}{2 \kappa^{2}} \int\left(R * \mathbf{1}-\frac{1}{2(\operatorname{Im} \tau)^{2}} d \tau \wedge * d \bar{\tau}\right),
$$

where $\kappa^{2}$ is the ten-dimensional gravitational constant, $*$ is the Hodge dual operator in the ten-dimensional spacetime, and $\tau=C_{(0)}+i e^{-\phi}$. The expectation values of fermionic fields 
are assumed to be zero. We can write the field equations by using the ten-dimensional action (89),

$$
\begin{aligned}
& R_{M N}=\frac{1}{(\operatorname{Im} \tau)^{2}}\left(\partial_{M} \tau \partial_{N} \bar{\tau}+\partial_{M} \bar{\tau} \partial_{N} \tau\right) \\
& \triangle \tau=\frac{1}{i(\operatorname{Im} \tau)} g^{M N} \partial_{M} \tau \partial_{N} \tau
\end{aligned}
$$

where $\triangle$ is the ten-dimensional Laplace operator.

We adopt the following ansatz for the ten-dimensional metric:

$$
d s^{2}=q_{\mu \nu}(\mathrm{X}) d x^{\mu} d x^{\nu}+e^{\Psi(x, y)} \delta_{i j}(\mathrm{Y}) d y^{i} d y^{j},
$$

where $q_{\mu \nu}$ is a eight-dimensional metric which depends only on the eight-dimensional coordinates $x^{\mu}$, and $y^{i}$ denote the two-dimensional coordinates.

We first consider the Einstein Eqs. (90). Using the assumption (92), the Einstein equations are given by

$$
\begin{aligned}
& R_{\mu \nu}(\mathrm{X})-D_{\mu} D_{\nu} \Psi-\frac{1}{2} \partial_{\mu} \Psi \partial_{\nu} \Psi-\frac{1}{4(\operatorname{Im} \tau)^{2}}\left(\partial_{\mu} \tau \partial_{\nu} \bar{\tau}+\partial_{\mu} \bar{\tau} \partial_{\nu} \tau\right)=0, \\
& \delta_{i j} e^{\Psi}\left(\triangle_{\mathrm{X}} \Psi+q^{\rho \sigma} \partial_{\rho} \Psi \partial_{\sigma} \Psi\right)+\delta_{i j} \triangle_{\mathrm{Y}} \Psi+\frac{1}{2(\operatorname{Im} \tau)^{2}}\left(\partial_{i} \tau \partial_{j} \bar{\tau}+\partial_{i} \bar{\tau} \partial_{j} \tau\right)=0, \\
& \partial_{\mu} \partial_{i} \Psi+\frac{1}{2(\operatorname{Im} \tau)^{2}}\left(\partial_{\mu} \tau \partial_{i} \bar{\tau}+\partial_{\mu} \bar{\tau} \partial_{i} \tau\right)=0,
\end{aligned}
$$

where $D_{\mu}$ is the covariant derivative with respect to the metric $q_{\mu \nu}(\mathrm{X}), \triangle_{\mathrm{X}}$ and $\triangle_{\mathrm{Y}}$ are the Laplace operators on the space of $\mathrm{X}$ and the space of $\mathrm{Y}$, and $R_{\mu \nu}(\mathrm{X})$ is the Ricci tensors of the metrics $q_{\mu \nu}$, respectively.

Now we introduce the complex coordinate $z=y^{1}+i y^{2}$ and assume that the scalar field $\tau$ depends only on the coordinate $z$ and $\bar{z}[29]$

$$
\tau=\tau(z, \bar{z})
$$

Then, from Eq. (95), the warp factor $\Psi$ must be in the form

$$
\Psi(x, z, \bar{z})=\Psi_{0}(x)+\Psi_{1}(z, \bar{z}) .
$$

Using the form of $\Psi$ and the assumption (96), the field equations Eqs. (93) and (94) are rewritten as

$$
\begin{aligned}
& R_{\mu \nu}(\mathrm{X})-D_{\mu} D_{\nu} \Psi_{0}-\frac{1}{2} \partial_{\mu} \Psi_{0} \partial_{\nu} \Psi_{0}=0 \\
& q^{\rho \sigma}(\mathrm{X})\left(D_{\rho} D_{\sigma} \Psi_{0}+\partial_{\rho} \Psi_{0} \partial_{\sigma} \Psi_{0}\right)=0 \\
& \partial \bar{\partial} \Psi_{1}-\partial \bar{\partial} \ln (\operatorname{Im} \tau)=0
\end{aligned}
$$


where $\partial, \bar{\partial}$ are defined by

$$
\partial=\frac{\partial}{\partial z} \equiv \frac{1}{2}\left(\frac{\partial}{\partial y^{1}}-i \frac{\partial}{\partial y^{2}}\right), \quad \bar{\partial}=\frac{\partial}{\partial \bar{z}} \equiv \frac{1}{2}\left(\frac{\partial}{\partial y^{1}}+i \frac{\partial}{\partial y^{2}}\right) .
$$

If the function $\Psi_{0}$ satisfies the equation,

$$
D_{\mu} D_{\nu} \Psi_{0}+\partial_{\mu} \Psi_{0} \partial_{\nu} \Psi_{0}=0
$$

Eq. (99) is satisfied and the field Eqs. (98) and (100) become

$$
\begin{aligned}
& R_{\mu \nu}(\mathrm{X})+\frac{1}{2} \partial_{\mu} \Psi_{0} \partial_{\nu} \Psi_{0}=R_{\mu \nu}(\mathrm{X})-\frac{1}{2} D_{\mu} D_{\nu} \Psi_{0}=0, \\
& \partial \bar{\partial} \Psi_{1}-\partial \bar{\partial} \ln (\operatorname{Im} \tau)=0 .
\end{aligned}
$$

If the eight-dimensional space $\mathrm{X}$ is assumed to be an Einstein manifold, Eq. (103) implies that the eight-dimensional metric $q_{\mu \nu}$ is expressed as a product of two vectors. Hence, the determinant of the metric $q_{\mu \nu}$ becomes zero, which is not permissible. It then follows that $R_{\mu \nu}(\mathrm{X})=0$ and $\Psi_{0}$ is constant, which implies that the function $\Psi$ depends only on the coordinates $z, \bar{z}$. That is, the solution is static. Static solutions of the field Eq. (104) were discussed in [14, 16, 17, 29]. The D7-brane solution (92) is different from the $p$-brane solutions (6) because the scalar field $\tau$ does not depend on the eight-dimensional coordinate $x^{\mu}$. To obtain a time-dependent solution, we will add a D3 brane, which we will discuss in the next subsection.

\section{B. D3-D7 brane solution}

We consider a gravitational theory with the metric $g_{M N}$, scalar field $\tau$, and a 5 -form field strength $F_{(5)}$. The action for the D3-D7 brane system in the Einstein frame can be written as

$$
S=\frac{1}{2 \kappa^{2}} \int\left(R * \mathbf{1}-\frac{1}{2(\operatorname{Im} \tau)^{2}} d \tau \wedge * d \bar{\tau}-\frac{1}{4} F_{(5)} \wedge * F_{(5)}\right),
$$

where $\kappa^{2}$ is the ten-dimensional gravitational constant and $*$ is the Hodge dual operator in the ten-dimensional spacetime. The expectation values of fermionic fields are assumed to be zero. 
The ten-dimensional action (105) gives following field equations:

$$
\begin{aligned}
& R_{M N}=\frac{1}{4(\operatorname{Im} \tau)^{2}}\left(\partial_{M} \tau \partial_{N} \bar{\tau}+\partial_{M} \bar{\tau} \partial_{N} \tau\right)+\frac{1}{96} F_{M A B C D} F_{N}{ }^{A B C D}, \\
& \triangle \tau=\frac{1}{i(\operatorname{Im} \tau)} g^{M N} \partial_{M} \tau \partial_{N} \tau \\
& d F_{(5)}=0, \quad F_{(5)}=* F_{(5)},
\end{aligned}
$$

where $\triangle$ is the ten-dimensional Laplace operator, and we used the self-duality condition for the 5 -form field strength, which is required by supersymmetry [28], and $\triangle$ is the tendimensional Laplace operator.

We assume the form of the ten-dimensional metric as

$$
\begin{aligned}
d s^{2} & =g_{M N} d x^{M} d x^{N} \\
& =h^{-1 / 2}(x, y) q_{\mu \nu}(\mathrm{X}) d x^{\mu} d x^{\nu}+h^{1 / 2}(x, y) u_{i j}(\mathrm{Y}) d y^{i} d y^{j}
\end{aligned}
$$

where $q_{\mu \nu}(\mathrm{X})$ denotes a four-dimensional metric, which depends only on the four-dimensional coordinates $x^{\mu}$, and $u_{i j}(\mathrm{Y})$ is the six-dimensional metric, which depends only on the sixdimensional coordinates $y^{i}$. The brane configuration is given as follows:

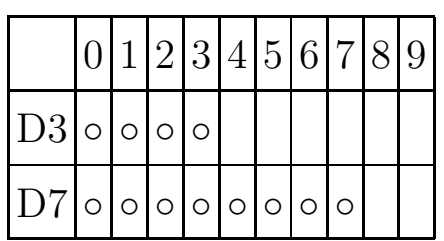

Furthermore, we assume that the scalar field $\tau$ and the gauge field strength $F_{5}$ are given by

$$
\begin{aligned}
\tau & =\tau(y), \\
F_{(5)} & =(1 \pm *) d\left(h^{-1}\right) \wedge \Omega(\mathrm{X}),
\end{aligned}
$$

where $\Omega(\mathrm{X})$ denotes the volume 4-form

$$
\Omega(\mathrm{X})=\sqrt{-q} d x^{0} \wedge d x^{1} \wedge d x^{2} \wedge d x^{3}
$$

Here, $q$ is the determinant of the metric $q_{\mu \nu}(\mathrm{X})$.

Now we assume that the metric $u_{i j}(\mathrm{Y})$ is given by

$$
u_{i j}(\mathrm{Y}) d y^{i} d y^{j}=s_{a b}(\mathrm{Y}) d w^{a} d w^{b}+e^{\Psi(r)}\left(d r^{2}+r^{2} d \theta^{2}\right)
$$


where $s_{a b}(\mathrm{Y})$ is the metric of the four-dimensional space, and $\Psi$ is a function that depends only on the coordinate $r$.

We adopt an assumption for the scalar field $\tau$ and the function $\Psi$ as[17, 29]

$$
\begin{aligned}
\tau & =C_{(0)}+i e^{-\phi} ; \\
C_{(0)} & =A \theta, \\
e^{-\phi(r)} & =e^{\Psi(r)}=-A \ln \left(\frac{r}{r_{\mathrm{c}}}\right),
\end{aligned}
$$

where $A$ and $r_{\mathrm{c}}$ are constant parameters. We note that, in this case, the square of the Ricci tensor for the internal space (113) is given by

$$
R^{i j}(\mathrm{Y}) R_{i j}(\mathrm{Y})=\frac{1}{2 r^{4}}\left[A \ln \left(\frac{r}{r_{\mathrm{c}}}\right)\right]^{-6} .
$$

Thus, the six-dimensional space $\mathrm{Y}$ has singularities at $r=0$ and $r=r_{\mathrm{c}}$.

Let us first consider the Einstein Eqs. (106). Using the assumptions (109), (110), (111), (113), the Einstein equations are given by

$$
\begin{aligned}
& R_{\mu \nu}(\mathrm{X})-h^{-1} D_{\mu} D_{\nu} h+\frac{1}{4} h^{-1} q_{\mu \nu}\left(\triangle_{\mathrm{X}} h+h^{-1} \triangle_{\mathrm{Y}} h\right)=0 \\
& R_{i j}(\mathrm{Y})-\frac{1}{4} u_{i j}\left(\triangle_{\mathrm{X}} h+h^{-1} \triangle_{\mathrm{Y}} h\right)=\frac{1}{4(\operatorname{Im} \tau)^{2}}\left(\partial_{i} \tau \partial_{j} \bar{\tau}+\partial_{i} \bar{\tau} \partial_{j} \tau\right), \\
& \partial_{\mu} \partial_{i} h=0
\end{aligned}
$$

where $D_{\mu}$ is the covariant derivative with respect to the metric $q_{\mu \nu}, \triangle_{\mathrm{X}}$ and $\triangle_{\mathrm{Y}}$ are the Laplace operators on the space of $\mathrm{X}$ and the space $\mathrm{Y}$, respectively, and $R_{\mu \nu}(\mathrm{X})$ and $R_{i j}(\mathrm{Y})$ are the Ricci tensors of the metrics $q_{\mu \nu}$ and $u_{i j}$, respectively. From Eq. (119), the warp factor $h$ must be in the form

$$
h(x, y)=h_{0}(x)+h_{1}(y)
$$

Using (120), Eqs. (117) and (118) are rewritten as

$$
\begin{aligned}
& R_{\mu \nu}(\mathrm{X})-h^{-1} D_{\mu} D_{\nu} h_{0}+\frac{1}{4} h^{-1} q_{\mu \nu}\left(\triangle_{\mathrm{X}} h_{0}+h^{-1} \triangle_{\mathrm{Y}} h_{1}\right)=0, \\
& R_{i j}(\mathrm{Y})-\frac{1}{4} u_{i j}\left(\triangle_{\mathrm{X}} h_{0}+h^{-1} \triangle_{\mathrm{Y}} h_{1}\right)=\frac{1}{4(\operatorname{Im} \tau)^{2}}\left(\partial_{i} \tau \partial_{j} \bar{\tau}+\partial_{i} \bar{\tau} \partial_{j} \tau\right) .
\end{aligned}
$$

In order to simplify these equations, we use the gauge and scalar field equations.

First we consider the gauge field equation. Under the assumption (111), we find

$$
d F_{(5)}=\mp \triangle_{Y} h_{1} d y^{i} \wedge *_{Y} d y^{i}=0,
$$


where we have used (120), and $*_{\text {Y }}$ denotes the Hodge dual operator on Y. Thus, we obtain the equation,

$$
\triangle_{\mathrm{Y}} h_{1}=0 .
$$

Next we consider the scalar field equation. Substituting the form of the scalar field, (114) and (115), and the metric (109) with (113) into the equation of motion for the scalar field $\tau$, (107), one finds it is automatically satisfied.

Then, noting the fact that $\tau$ is a function of only the coordinates $y^{i}$, Eq. (122) implies $\triangle_{\mathrm{X}} h_{0}=0$ if the function $h_{1}$ is nontrivial. In this case, the Einstein equations reduce to

$$
\begin{aligned}
& R_{\mu \nu}(\mathrm{X})=0, \\
& R_{a b}(\mathrm{Y})=0, \\
& D_{\mu} D_{\nu} h_{0}=0,
\end{aligned}
$$

where $R_{a b}(\mathrm{Y})$ is the Ricci tensor with respect to the metric $s_{a b}(\mathrm{Y})$. The Einstein equations with respect to $R_{r r}, R_{r \theta}, R_{\theta \theta}$ are automatically satisfied under the assumptions (113), (114), (115). If the function $h_{1}$ is trivial, that is, if it is a constant, we may set $h_{1}=0$ without loss of generality. This means we have $F_{(5)}=0$ and $h=h_{0}(x)$. It then follows from (122) that $\triangle_{\mathrm{X}} h_{0}=0$. If we assume $q_{\mu \nu}=\eta_{\mu \nu}, s_{a b}=\delta_{a b}$, the solution for $h_{0}$ is given by

$$
h_{0}(x)=c_{\mu} x^{\mu}+d,
$$

in terms of the four-dimensional Minkowski coordinates $x^{\mu}$, where $c_{\mu}$ and $d$ are constants.

Equation (126) implies that the function $h_{0}$ is in the same form as in the case of a single D3-brane solution with $\lambda=0$ as seen from Eq. (28). Thus, we find that the metric for the D3-D7 brane system (109) is similar to that of the D3-brane system. Apart from the restriction that $\lambda=0$, the difference from the D3-brane metric is in the six-dimensional metric $u_{i j}$, which is affected by the existence of a nontrivial scalar field configuration, which describes a D7 brane.

The ten-dimensional metric (109) exists for $h>0$ and has curvature singularities at $h=0, r=0$, and $r=r_{\mathrm{c}}$. If the function $h_{1}$ is negligible and $h_{0}(t) \propto t$, the scale factor of the four-dimensional universe is $\propto \tau^{-2 / 3}$, while the scale factor of the six-dimensional internal space is $\propto \tau^{2 / 3}$, where $\tau \propto t^{3 / 4}$ is the cosmic time in four dimensions. Thus, as in the case of the D4-D8 brane system, the D3-D7 solution also behaves as a Kaluza-Klein 
type cosmological solution in the asymptotic future. On the other hand, the analysis of the solution near $t=0$ is not so easy compared to the case of the D4-D8 brane system. Therefore, we postpone it as a future issue.

We can construct the D3-D7-brane solution on the basis of the discussion in Sec. II. Now we assume the ten-dimensional metric of the form

$$
\begin{aligned}
d s^{2}= & h_{3}^{-1 / 2}(x, y) q_{\mu \nu}(\mathrm{X}) d x^{\mu} d x^{\nu}+h_{3}^{1 / 2}(x, y) \gamma_{a b}(\mathrm{Z}) d z^{a} d z^{b} \\
& +h_{3}^{1 / 2}(x, y) h_{7}(x, y) u_{i j}(\mathrm{Y}) d y^{i} d y^{j}
\end{aligned}
$$

where $q_{\mu \nu}(\mathrm{X})$ denotes a four-dimensional metric, which depends only on the four-dimensional coordinates $x^{\mu}, \gamma_{a b}(Z)$ denotes a four-dimensional metric, which depends only on the fourdimensional coordinates $z^{a}$, and $u_{i j}(\mathrm{Y})$ is the two-dimensional metric, which depends only on the two-dimensional coordinates $y^{i}$. We also assume that the dilaton $\phi$ and the gauge fields are given by

$$
\begin{aligned}
F_{(5)} & =(1 \pm *) d\left(h_{5}^{-1}\right) \wedge \Omega(\mathrm{X}), \\
F_{(1)} & =e^{-2 \phi} * F_{(9)}=e^{-2 \phi} *\left[d\left(h_{7}^{-1}\right) \wedge \Omega(\mathrm{X}) \wedge \Omega(\mathrm{Z})\right], \\
e^{\phi} & =h_{7}^{-1},
\end{aligned}
$$

where $\Omega(\mathrm{X})$ and $\Omega(\mathrm{Z})$ are given by

$$
\Omega(\mathrm{X})=\sqrt{-q} d x^{0} \wedge \cdots \wedge d x^{3}, \quad \Omega(\mathrm{Z})=\sqrt{\gamma} d z^{4} \wedge \cdots \wedge d z^{7}
$$

The dilaton and gauge fields are assumed to have the same form as the single $p$-brane solutions in Sec. [I]. The assumptions on the ten-dimensional metric and fields correspond to the following brane configuration:

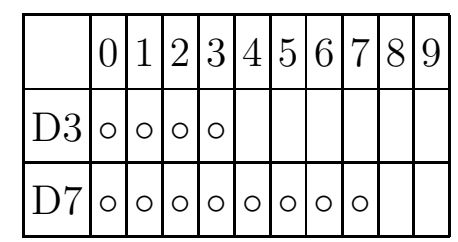

This is the same as the previous D3-D7-brane configuration (109). We first consider the Einstein Eqs. (106). Substituting the metric (128) into the ten-dimensional Einstein equations, 
we obtain

$$
\begin{aligned}
& R_{\mu \nu}(\mathrm{X})-h_{3}^{-1} D_{\mu} D_{\nu} h_{3}-h_{7}^{-1} D_{\mu} D_{\nu} h_{7}-\frac{1}{2}\left(\partial_{\mu} \ln h_{3} \partial_{\nu} \ln h_{7}+\partial_{\mu} \ln h_{7} \partial_{\nu} \ln h_{3}\right) \\
& \quad+\frac{1}{4} h_{3}^{-1} q_{\mu \nu}\left[\triangle_{\mathrm{X}} h_{3}+\left(h_{3} h_{7}\right)^{-1}\left(\triangle_{\mathrm{Y}} h_{3}+h_{3} q^{\rho \sigma} \partial_{\rho} h_{3} \partial_{\sigma} h_{7}\right)\right]=0, \\
& R_{a b}(\mathrm{Z})-\frac{1}{4} \gamma_{a b}\left[\triangle_{\mathrm{X}} h_{3}+\left(h_{3} h_{7}\right)^{-1}\left(\triangle_{\mathrm{Y}} h_{3}+h_{3} q^{\rho \sigma} \partial_{\rho} h_{3} \partial_{\sigma} h_{7}\right)\right]=0, \\
& R_{i j}(\mathrm{Y})-\frac{1}{4} u_{i j}\left(h_{7} \triangle_{\mathrm{X}} h_{3}+h_{3}^{-1} \triangle_{\mathrm{Y}} h_{3}\right)-\frac{1}{2} u_{i j}\left(h_{3} \triangle_{\mathrm{X}} h_{7}+h_{7}^{-1} \triangle_{\mathrm{Y}} h_{7}\right) \\
& \quad-\frac{3}{4} u_{i j} q^{\rho \sigma} \partial_{\rho} h_{3} \partial_{\sigma} h_{7}=0, \\
& h_{3}^{-1} \partial_{\mu} \partial_{i} h_{3}+h_{7}^{-1} \partial_{\mu} \partial_{i} h_{7}=0,
\end{aligned}
$$

where $D_{\mu}$ is the covariant derivative with respect to the metric $q_{\mu \nu}, \triangle_{\mathrm{X}}$ and $\triangle_{\mathrm{Y}}$ are the Laplace operators on the space of $\mathrm{X}$ and the space $\mathrm{Y}$, respectively, and $R_{\mu \nu}(\mathrm{X}), R_{a b}(\mathrm{Z})$, and $R_{i j}(\mathrm{Y})$ are the Ricci tensors of the metrics $q_{\mu \nu}, \gamma_{a b}$, and $u_{i j}$, respectively. From Eq. (136), we have

$$
h_{3}(x, y)=H_{0}(x)+H_{1}(y), \quad h_{7}(x, y)=K_{0}(x)+K_{1}(y) .
$$

Using (137), Eqs. (133), (134) and (135) are rewritten as

$$
\begin{aligned}
& R_{\mu \nu}(\mathrm{X})-h_{3}^{-1} D_{\mu} D_{\nu} H_{0}-h_{7}^{-1} D_{\mu} D_{\nu} K_{0}-\frac{1}{2}\left(h_{3} h_{7}\right)^{-1}\left(\partial_{\mu} H_{0} \partial_{\nu} K_{0}+\partial_{\mu} K_{0} \partial_{\nu} H_{0}\right) \\
& \quad+\frac{1}{4} h_{3}^{-1} q_{\mu \nu}\left[\triangle_{\mathrm{X}} H_{0}+\left(h_{3} h_{7}\right)^{-1}\left(\triangle_{\mathrm{Y}} H_{1}+h_{3} q^{\rho \sigma} \partial_{\rho} H_{0} \partial_{\sigma} K_{0}\right)\right]=0 \\
& R_{a b}(\mathrm{Z})-\frac{1}{4} \gamma_{a b}\left[\triangle_{\mathrm{X}} H_{0}+\left(h_{3} h_{7}\right)^{-1}\left(\triangle_{\mathrm{Y}} H_{1}+h_{3} q^{\rho \sigma} \partial_{\rho} H_{0} \partial_{\sigma} K_{0}\right)\right]=0 \\
& R_{i j}(\mathrm{Y})-\frac{1}{4} u_{i j}\left(h_{7} \triangle_{\mathrm{X}} H_{0}+h_{3}^{-1} \triangle_{\mathrm{Y}} H_{1}\right)-\frac{1}{2} u_{i j}\left(h_{3} \triangle_{\mathrm{X}} K_{0}+h_{7}^{-1} \triangle_{\mathrm{Y}} K_{1}\right) \\
& \quad-\frac{3}{4} u_{i j} q^{\rho \sigma} \partial_{\rho} H_{0} \partial_{\sigma} K_{0}=0 .
\end{aligned}
$$

We can simplify Eqs. (138) -(140) in terms of the gauge and dilaton equations. Under the assumption (129), we find

$$
d F_{(5)}=\mp \triangle_{\mathrm{Y}} H_{1} \Omega(\mathrm{Z}) \wedge d y^{i} \wedge *_{\mathrm{Y}} d y^{i}=0,
$$

where we have used (137), and $*_{Y}$ denotes the Hodge dual operator on Y. Thus, we obtain the equation,

$$
\triangle_{\mathrm{Y}} H_{1}=0
$$

The scalar field Eq. (107) can be decomposed as follows

$$
\begin{aligned}
& d\left(e^{2 \phi} * F_{(1)}\right)=0, \\
& \triangle \phi=e^{2 \phi} F_{(1)}^{2} .
\end{aligned}
$$


Under Eq. (130), the field Eq. (143) becomes the Bianchi identity for $F_{(9)}$. Then, the field equation for $F_{(1)}$ is automatically satisfied. On the other hand, from the Bianchi identity for $F_{(1)}$, we have

$$
d\left(e^{-2 \phi} * F_{(9)}\right)=-\triangle_{\mathrm{Y}} K_{1} d y^{i} \wedge *_{\mathrm{Y}} d y^{i}=0 .
$$

Then, the Bianchi identity for $F_{(1)}$ leads to

$$
\triangle_{\mathrm{Y}} K_{1}=0
$$

Next we consider the dilaton Eq. (144). Substituting Eqs. (129) - (131), and the metric (128) into the equation of motion for the dilaton (144), we find

$$
h_{3}^{-1 / 2} h_{7}^{-1}\left(q^{\mu \nu} \partial_{\mu} H_{0} \partial_{\nu} K_{0}+h_{3} \triangle_{\mathrm{X}} H_{0}\right)=0
$$

where we used Eqs. (137), (146). Then, in order to satisfy field Eqs. (138) - (140), (142), (146), (147), we can choose either

$$
\begin{aligned}
& R_{\mu \nu}(\mathrm{X})=0, \quad R_{a b}(\mathrm{Z})=0, \quad R_{i j}(\mathrm{Y})=0 \\
& D_{\mu} D_{\nu} H_{0}=0, \quad K_{0}=\text { constant }
\end{aligned}
$$

or

$$
\begin{aligned}
& R_{\mu \nu}(\mathrm{X})=0, \quad R_{a b}(\mathrm{Z})=0, \quad R_{i j}(\mathrm{Y})=0, \\
& D_{\mu} D_{\nu} K_{0}=0, \quad H_{0}=\text { constant. }
\end{aligned}
$$

We find that the Einstein equations cannot be satisfied if both $h_{3}$ and $h_{7}$ depend on the coordinate $x^{\mu}$.

If we assume $\partial_{\mu} h_{7}=0, q_{\mu \nu}=\eta_{\mu \nu}, \gamma_{a b}=\delta_{a b}, u_{i j}=\delta_{i j}$, we have

$$
\begin{aligned}
& h_{3}(x, y)=H_{0}(x)+H_{1}(y) ; \quad H_{0}(x)=c_{\mu} x^{\mu}+d, \quad H_{1}(y)=\sum_{l} M_{l} \ln \left|\vec{y}-\vec{y}_{l}\right|, \\
& h_{7}(y)=\sum_{l} L_{l} \ln \left|\vec{y}-\vec{y}_{l}\right|,
\end{aligned}
$$

where $c_{\mu}, d, M_{l}, L_{l}$ and $\vec{y}_{l}$ are constants. For $\partial_{\mu} h_{3}=0, q_{\mu \nu}=\eta_{\mu \nu}, \gamma_{a b}=\delta_{a b}, u_{i j}=\delta_{i j}$, we have

$$
\begin{aligned}
& h_{7}(x, y)=K_{0}(x)+K_{1}(y) ; \quad K_{0}(x)=c_{\mu} x^{\mu}+d, \quad K_{1}(y)=\sum_{l} M_{l} \ln \left|\vec{y}-\vec{y}_{l}\right|, \\
& h_{3}(y)=\sum_{l} L_{l} \ln \left|\vec{y}-\vec{y}_{l}\right|,
\end{aligned}
$$


where $c_{\mu}, d, M_{l}, L_{l}$, and $\vec{y}_{l}$ are constants.

There are curvature singularities at $\vec{y}=\vec{y}_{l}$ as well as at $h_{3}=0$ or $h_{7}=0$ in the tendimensional metric (128). In the case of $\partial_{\mu} h_{7}=0$, the function $H_{0}$ depends on a linear function of the four-dimensional coordinates $x^{\mu}$. The scale factor of the four-dimensional universe has the same form as the previous D3-D7-brane solution (109). For $\partial_{\mu} h_{3}=0$, the function $K_{0}$ is proportional to a linear function of the four-dimensional coordinates $x^{\mu}$. The scale factor $a(t)$ of the two-dimensional internal space $\mathrm{Y}$ is given by $a(t) \propto t$ if $K_{1}=0$ and $K_{0}(t) \propto t$.

The D3-D7-brane metric (128) is not the same as (109) because it has the dilaton and gauge field, which depend on the four-dimensional coordinate $x^{\mu}$. In particular, the twodimensional internal space $\mathrm{Y}$ depends on time even if $\partial_{\mu} h_{3}=0$.

\section{CONCLUSION}

In this paper, we investigated dynamical solutions of higher-dimensional supergravity models. We found a class of time-dependent solutions for intersecting D4-D8- and D3D7-brane systems. These solutions were obtained by replacing a constant $A$ in the warp factor $h=A+h_{1}(y)$ of a supersymmetric solution by a function $h_{0}(x)$ of the coordinates $x^{\mu}$ [21], where the coordinates $y^{i}$ would describe the internal space and $x^{\mu}$ would describe our Universe. In the D4-D8 brane solution, the geometry was found to approach a warped static $\mathrm{AdS}_{6} \times \mathrm{S}^{4}$ in a certain region of the spacetime.

In particular, in the case of the D4-D8 system, we found an interesting solution which is warped and static as $\tau \rightarrow 0$ but approaches a Kasner-type solution as $\tau \rightarrow \infty$, where $\tau$ is the cosmic time. Although the solution itself is by no means realistic, its interesting behavior suggests a possibility that the Universe was originally in a static state of warped compactification and began to evolve toward a Universe with a Kaluza-Klein compactified internal space.

Conventionally one would expect an effective theory description in lower dimensions to be valid at low energies. However, as clearly the case of the cosmological solution mentioned above, the solutions we found have the property that they are genuinely $D$ dimensional in the sense that one can never neglect the dependence on $y^{i}$, say of $h$. Thus, our result indicates that we have to be careful when we use a four-dimensional effective theory to 
analyze the moduli stabilization problem and the cosmological problems in the framework of warped compactification of supergravity or M theory.

\section{Acknowledgments}

This work was supported by the CNRS-JSPS Joint Research Program and by JSPS Grantin-Aid for Scientific Research (B), under Contract Nos. 17340075 and (A) 18204024, and also by JSPS Grant-in-Aid for Creative Scientific Research, under Contract No. 19GS0219. $\mathrm{KU}$ is supported by the Kwansei Gakuin University, Grant-in-Aid for Young Scientists (B) of JSPS Research, under Contract No. 20740147, and he is grateful to Shigeki Sugimoto for valuable discussions.

\section{References}

[1] H. Kodama and K. Uzawa, "Comments on the four-dimensional effective theory for warped compactification," JHEP 0603 (2006) 053 arXiv:hep-th/0512104.

[2] G. W. Gibbons, H. Lu and C. N. Pope, "Brane worlds in collision," Phys. Rev. Lett. 94 (2005) 131602 arXiv:hep-th/0501117.

[3] M. J. Duff, B. E. W. Nilsson and C. N. Pope, "Kaluza-Klein supergravity," Phys. Rept. 130 (1986) 1.

[4] N. Ohta and K. L. Panigrahi, "Supersymmetric intersecting branes in time-dependent backgrounds," Phys. Rev. D 74 (2006) 126003 arXiv:hep-th/0610015].

[5] R. Güven, "Black p-brane solutions of D = 11 supergravity theory," Phys. Lett. B 276 (1992) 49.

[6] J. Polchinski and E. Witten, "Evidence for heterotic - type I string duality," Nucl. Phys. B 460 (1996) 525 arXiv:hep-th/9510169.

[7] D. Youm, "Localized intersecting BPS branes," arXiv:hep-th/9902208.

[8] N. Ohta, "Intersection rules for non-extreme p-branes," Phys. Lett. B 403 (1997) 218 arXiv:hep-th/9702164.

[9] H. Nastase, "On Dp-Dp+4 systems, QCD dual and phenomenology," arXiv:hep-th/0305069. 
[10] A. Brandhuber and Y. Oz, "The D4-D8 brane system and five dimensional fixed points," Phys. Lett. B 460 (1999) 307 arXiv:hep-th/9905148.

[11] L. J. Romans, "Massive N=2a supergravity in ten-dimensions," Phys. Lett. B 169 (1986) 374.

[12] L. J. Romans, "The F(4) gauged supergravity in six-dimensions," Nucl. Phys. B 269 (1986) 691.

[13] M. Cvetic, H. Lu and C. N. Pope, "Gauged six-dimensional supergravity from massive type IIA," Phys. Rev. Lett. 83 (1999) 5226 arXiv:hep-th/9906221].

[14] O. Aharony, A. Fayyazuddin and J. M. Maldacena, "The large N limit of $\mathrm{N}=2,1$ field theories from three-branes in F-theory," JHEP 9807 (1998) 013 [arXiv:hep-th/9806159].

[15] M. Grana and J. Polchinski, "Gauge / gravity duals with holomorphic dilaton," Phys. Rev. D 65 (2002) 126005 [arXiv:hep-th/0106014].

[16] B. A. Burrington, J. T. Liu, L. A. Pando Zayas and D. Vaman, "Holographic duals of flavored N = 1 super Yang-Mills: Beyond the probe approximation," JHEP 0502 (2005) 022 arXiv:hep-th/0406207.

[17] I. Kirsch and D. Vaman, "The D3/D7 background and flavor dependence of Regge trajectories," Phys. Rev. D 72 (2005) 026007 arXiv:hep-th/0505164.

[18] H. Lu, C. N. Pope, E. Sezgin and K. S. Stelle, "Stainless super p-branes," Nucl. Phys. B 456 (1995) 669 arXiv:hep-th/9508042.

[19] K. S. Stelle, "BPS branes in supergravity," arXiv:hep-th/9803116.

[20] R. Argurio, "Brane physics in M-theory," arXiv:hep-th/9807171.

[21] H. Kodama and K. Uzawa, "Moduli instability in warped compactifications of the type IIB supergravity," JHEP 0507 (2005) 061 arXiv:hep-th/0504193.

[22] E. Bergshoeff, M. de Roo, M. B. Green, G. Papadopoulos and P. K. Townsend, "Duality of Type II 7-branes and 8-branes," Nucl. Phys. B 470 (1996) 113 arXiv:hep-th/9601150.

[23] E. Bergshoeff, Y. Lozano and T. Ortin, "Massive branes," Nucl. Phys. B 518 (1998) 363 arXiv:hep-th/9712115].

[24] Y. Imamura, "1/4 BPS solutions in massive IIA supergravity," Prog. Theor. Phys. 106 (2001) 653 arXiv:hep-th/0105263.

[25] M. Cvetic, S. S. Gubser, H. Lu and C. N. Pope, "Symmetric potentials of gauged supergravities in diverse dimensions and Coulomb branch of gauge theories," Phys. Rev. D 62 (2000) 086003 arXiv:hep-th/9909121. 
[26] K. Behrndt, E. Bergshoeff, R. Halbersma and J. P. van der Schaar, "On domain-wall/QFT dualities in various dimensions," Class. Quant. Grav. 16 (1999) 3517 [arXiv:hep-th/9907006].

[27] C. Nunez, I. Y. Park, M. Schvellinger and T. A. Tran, "Supergravity duals of gauge theories from $\mathrm{F}(4)$ gauged supergravity in six dimensions," JHEP 0104 (2001) 025 arXiv:hep-th/0103080].

[28] J. H. Schwarz, "Covariant field equations of chiral N=2 D=10 supergravity," Nucl. Phys. B 226 (1983) 269.

[29] B. R. Greene, A. D. Shapere, C. Vafa and S. T. Yau, "Stringy cosmic strings and noncompact Calabi-Yau manifolds," Nucl. Phys. B 337 (1990) 1. 